BULLETIN OF THE

AMERICAN MATHEMATICAL SOCIETY

Volume 79, Number 2, March 1973

\title{
PRIME IDEALS IN NOETHERIAN PI-RINGS
}

BY LANCE W. SMALL

Communicated by Saunders Mac Lane, September 11, 1972

1. One of the more striking features of commutative Noetherian rings is the fact that they satisfy the descending chain condition on prime ideals. It is known that arbitrary right Noetherian rings need not enjoy this property [3]. In this note we show that if $R$ is a ring satisfying a polynomial identity and the ascending chain condition on two-sided ideals, then $R$ satisfies the descending chain condition on prime ideals.

Throughout this note all rings will have a unit element and satisfy a proper polynomial identity over some commutative ring. The word "ideal" will mean two-sided ideal.

2. If $R$ is a prime ring and $d$ is the minimal degree of the identities satisfied by $R$, then $d=2 n$ (for some $n \geqq 1$ ) and $R$ satisfies the standard identity $S_{2 n}$, see [1]. We call the integer $d$ the degree of $R$.

If $R$ is a prime ring of degree $d$ and $0 \neq P$ is a prime ideal in $R$ such that $R / P$ also has degree $d$, we showed in [5] that $R$ may be localized classically at the set $S=\{c \in R \mid c+P$ regular in $R / P\}$. The resulting ring $R_{S}$ has a unique maximal ideal, $P R_{S}$, and $R_{S} / P R_{S}$ also has degree $d$. Therefore, by a theorem of M. Artin [2] (see [4] for an extension to rings), $R_{S}$ is an Azumaya algebra of rank $(d / 2)^{2}$ over its center.

The pieces are now available to prove the

THEOREM. If $R$ is a PI-ring with the ascending chain condition on ideals, then $R$ satisfies the descending chain condition on prime ideals.

Proof. If $P_{1} \supsetneq P_{2} \supsetneq \cdots \supsetneq P_{n} \supsetneq \cdots$ is an infinite descending chain of prime ideals in $R$, then $V=\bigcap P_{i}$ is again a prime ideal. $\bar{R}=R / V$ is, thus, a prime ring with the ascending chain condition on ideals and with an infinite descending chain of primes $\bar{P}_{1} \supsetneq \bar{P}_{2} \supsetneq \cdots \supsetneq \bar{P}_{n} \supsetneq \cdots$ such that $\bigcap \bar{P}_{i}=0$. If $d=2 n$ is the degree of $\bar{R}$, there exist elements $\bar{r}_{1}, \ldots$, $\bar{r}_{2 n-2}$ such that $S_{2 n-2}\left[\bar{r}_{1}, \ldots, \bar{r}_{2 n-2}\right] \neq 0$ where $S_{2 n-2}$ is the standard polynomial of degree $2 n-2$. Therefore, since $\bigcap \bar{P}_{i}=0$, there is a $j$ such that $\bar{P}_{j}$ does not contain $S_{2 n-2}\left[\bar{r}_{1}, \ldots, \bar{r}_{2 n-2}\right]$. Hence, $R_{1}=\bar{R} / \bar{P}_{j}$ has the same degree as $\bar{R}$. Now form the localization $\bar{R}_{S}$ where $S$ is as above. Since $P_{i} \cap S=\varnothing$ for $i \geqq j$, the $P_{i} \bar{R}_{S}$ are distinct prime ideals in $\bar{R}_{S}$. However, $\bar{R}_{S}$ is an Azumaya algebra with the ascending chain condition on ideals. Hence, the center of $\bar{R}_{S}$ is Noetherian and, using the

AMS(MOS) subject classifications (1970). Primary 16A38, 16A46, 16A66. 
correspondence between ideals of $\bar{R}_{S}$ and those of its center, $\bar{R}_{S}$ satisfies the descending chain condition on prime ideals. Therefore, we have a contradiction and the theorem is proved.

\section{REFERENCES}

1. S. Amitsur, Prime rings having polynomial identities with arbitrary coefficients, Proc. London Math. Soc. (3) 17 (1967), 470-486. MR 36 \#209.

2. M. Artin, On Azumaya algebras and finite-dimensional representations of rings, $\mathbf{J}$. Algebra 11 (1969), 532-563. MR 39 \#4217.

3. A. Jategaonkar, A counter-example in ring theory and homological algebra, J. Algebra 12 (1969), 418-440. MR 39 \# 1485.

4. C. Procesi, On a theorem of M. Artin, J. Algebra 22 (1972), 309-315.

5. L. Small, Localization in PI-rings, J. Algebra 18 (1971), 269-270. MR 43 \#6265.

Department of Mathematics, University of California, San Diego, la Jolla, CALIFORNIA 92037. 